\title{
A Full-Wave Rectifier With Integrated Peak Selection for Multiple Electrode Piezoelectric Energy Harvesters
}

\author{
Nathaniel J. Guilar, Member, IEEE, Rajeevan Amirtharajah, Member, IEEE, and Paul J. Hurst, Fellow, IEEE
}

\begin{abstract}
Piezoelectric transducers are a viable way of harvesting vibrational energy for low power embedded systems such as wireless sensors. A proposed disk-shaped piezoelectric transducer with several electrodes enables increased energy harvesting from multiple mechanical resonances. To rectify the low-frequency AC voltage from harvested vibrational energy, a full-wave rectifier has been fabricated in $0.35 \mu \mathrm{m}$ CMOS. Integrated peak selection circuitry allows input signals with $90^{\circ}$ relative phase shift from the multiple-electrode piezoelectric transducer to be rectified with reduced output ripple. The rectifier has a measured power efficiency of $98.3 \%$ while delivering $90 \mu \mathrm{W}$ and occupying an area of $0.007 \mathrm{~mm}^{2}$. This high efficiency further enables energy harvesters to power wireless devices for extended durations.
\end{abstract}

Index Terms-Energy harvesting, piezoelectric, rectifier, wireless sensor.

\section{INTRODUCTION}

$\mathbf{E}$ NVIRONMENTAL energy harvesting has proven to be a viable way of extending wireless system lifetimes while reducing reliance on batteries. Depending on the environmental stimulus, solar, thermal gradients, and mechanical vibrations can be used as sources for energy scavenging [1]. Many vibration-based energy harvesting systems use a piezoelectric transducer as an AC power source. The generated AC voltage from the vibrational transducer must first be rectified before it is delivered to a load. In order to increase the system's energy harvesting ability over a wide range of vibrational frequencies, a multiple-electrode disk-shaped piezoelectric transducer has been proposed [2], [3]. Typically, the energy from a piezoelectric transducer that has two electrodes is rectified using a full-wave diode bridge rectifier [4], which requires a significant voltage drop between input and output, decreasing the rectifier's voltage efficiency. Previous works with rectifiers using MOS switches avoid the diode drop [5]-[13]; however, they lack the ability to efficiently rectify the multiple output phases generated from a disk-shaped transducer. In order to

Manuscript received April 13, 2008; revised June 19, 2008. Current version published December 24, 2008. This work was funded by UC MICRO Grant 06-206 and NSF CAREER Award \#0547113. The work of N. Guilar was supported by a U.S. Department of Education GAANN Fellowship.

N. J. Guilar is with Agilent Laboratories, Santa Clara, CA 95051 USA (e-mail: guilar@agilent.com).

R. Amirtharajah is with the Micropower Circuits and Systems Group, Department of Electrical and Computer Engineering, University of California, Davis, CA 95616-5270 USA

P. J. Hurst is with the Solid-State Circuits Research Laboratory, Department of Electrical and Computer Engineering, University of California, Davis, CA 95616-5270 USA.

Digital Object Identifier 10.1109/JSSC.2008.2007446

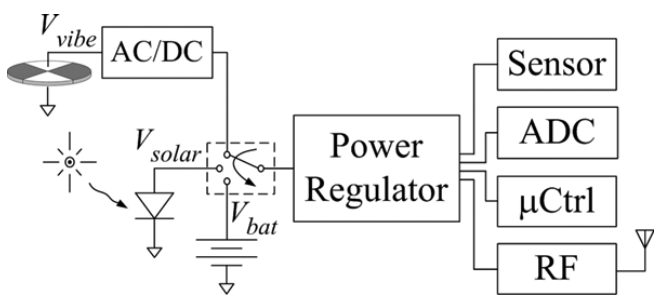

Fig. 1. A low power wireless system powered from energy scavengers and a battery. Energy sources are labeled $V_{\text {solar }}, V_{\text {vibe }}$ and $V_{b a t}$ for the solar, mechanical vibration and battery, respectively. A multiplexer switches between the unregulated energy sources.

increase the flexibility and efficiency of the rectifier, this paper describes a CMOS controlled rectifier (CCR) with integrated peak selection circuitry.

Fig. 1 shows a block diagram of an energy harvesting wireless sensor node system-on-chip powered by environmental sources [14]. Light and vibrations are converted to electrical energy by photodiodes and piezoelectric transducers, respectively. Environmental conditions determine the available system energy, which may change over time. A stored energy source such as a battery or ultracapacitor is also included to provide energy when environmental sources alone are insufficient. Additionally, the scavenged energy must be regulated before being used to power any load. Energy scalable algorithms can be used to vary the effective duty cycle for the system depending on the amount of available energy [15]. Each load may also present different requirements to the power regulator, such as specific voltage levels, output power, and maximum ripple. As these systems are capable of interacting with their environment, processing data, and communicating data to the outside world, they can be used for a variety of applications including medical monitoring, environmental sensing, and lighting, heating, and ventilation control for energy-efficient buildings. The particular energy transducers selected to power these devices may depend significantly on the intended application and expected environmental stimulus.

In this paper we focus on the transducer and the AC/DC conversion blocks shown in Fig. 1, where the AC/DC conversion is achieved using a full-wave MOS rectifier with internal peak selection circuitry, which selects the peak voltage generated from a multiple electrode piezoelectric transducer. The rest of the paper is organized as follows. Section II highlights the multiple-electrode piezoelectric disk as a generator for energy harvesting. Section III describes the principles of operation, design, and optimization of the proposed CCR. Results from testing an integrated circuit prototype of the rectifier implemented in 0.35 

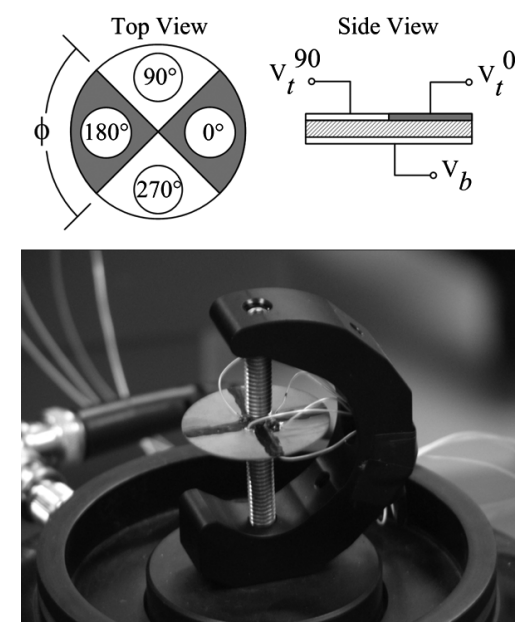

Fig. 2. Diagrams showing top and side views for the dual-layer piezoelectric disk, and picture of test setup with c-clamp and shaker.

$\mu \mathrm{m}$ CMOS are presented in Section IV, followed by conclusions.

\section{Piezoelectric TRANSDUCER}

Ultrasonic motors create rotary motion by exciting a traveling wave, which propagates around the circumference of a piezoelectric disk. Traditionally, the traveling wave is excited by stimulating a pattern of electrodes on the disk. Each electrode is driven by a signal which has a constant phase shift relative to the signals driving its neighboring electrodes [16]. In this work, we are relying on the input vibration to excite the traveling wave. Since piezoelectric transducers are bilateral devices, the generated electrical waveform measured at each electrode will have a similar phase relationship to its neighbors as the signals used to stimulate a traveling wave in a piezoelectric ultrasonic motor. This phase relationship between electrodes enables new, highly efficient interface circuits.

Since cantilever beam piezoelectric transducers can achieve high energy densities, they have been the focus for much research in vibration-based energy harvester design [17], [18]. However, many simple cantilever or membrane-based transducers are only efficient around a specific resonant frequency [7]. Due to the multiple electrode connections and free-free boundary conditions, the 2-layer piezoelectric disk transducers described here have multiple resonant frequencies. Each resonance for the disk is accompanied by a special modal shape [19], [20]. Since the vibrations seen by the energy harvesting system changes with environmental conditions, it is desirable to have a transducer that is responsive over a wide frequency range, and multiple resonant frequencies can help achieve this. With an input vibration at resonance, a disk shaped transducer will not be able to generate as much tip deflection as an equivalent sized cantilever transducer resulting in less power generation. However, with a random input vibration the multiple resonant frequencies of the disk may yield higher energy densities than a single resonance transducer.

Fig. 2 shows a photograph of the test fixture for the piezoelectric disk along with diagrams associated with the disk. At the bottom of the picture is a mechanical shaker, which is similar

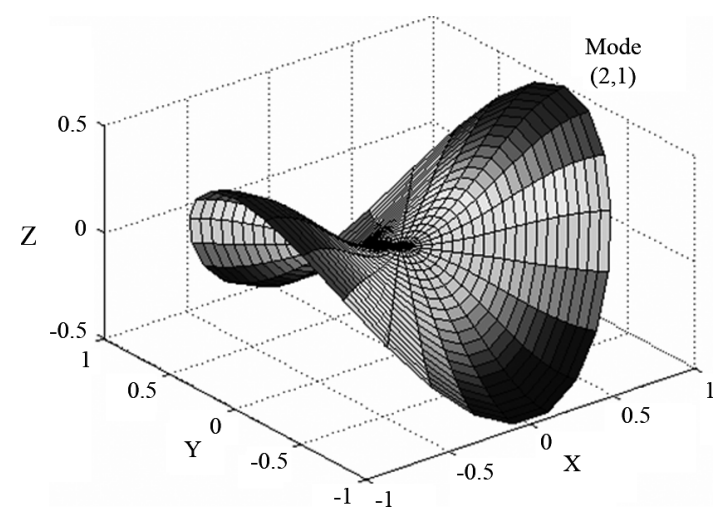

Fig. 3. Simulated traveling-wave mode shape for a thin plate.

to a voice coil in a speaker. Attached to the shaker is a c-clamp where threaded screws with nonconductive nylon tips support the dual-layer piezoelectric disk at its center. The top and side views of the disk show the multiple electrodes. Also indicated are the relative phase shifts $\phi$ of the electrode signals when the disk is vibrating in a traveling wave mode.

After initial fabrication, the top and bottom plates of the piezoelectric disk are intact. A file was used to score the disk on the top plate to form four quarter circle electrodes. This process results in four top-plate electrodes that are electrically isolated from each other but mechanically coupled through the disk. Including the bottom plate, there are a total of five electrodes. Leads were bonded to these electrodes with conductive epoxy. These connections were made towards the center of the disk to reduce mechanical stress on the epoxy bond.

Fig. 3 displays the results of a mathematical model of the disk's vibration with a traveling wave. In general, the vibrational pattern or mode shape will be a summation of modified and unmodified Bessel functions of the 1st and 2nd kind [21]. The vibrational mode shown is the traveling wave at $(2,1)$, where the mode number 2 corresponds to the number of modal diameters. If we trace around the edge of the disk, we will see two cycles of a sinusoid. The number 1 corresponds to the number of modal circumferences; a 1 here means the mode shape will continue in the same direction as one moves from the center to the edge of the disk, and the amplitude of the vibration will not cross zero again. The quarter circle electrodes proposed for our disk-shaped transducer would not be able to efficiently harvest energy from many higher frequency modes than the $(2,1)$ mode since for these configurations both positive and negative shear force exist in the same quarter-circle area. Additional radial cuts would be needed to harvest energy from these modes. However, the energy associated with higher frequency modes is usually small when compared to the lower frequency modes, and adding more cuts will decrease the mechanical strength of the disk, possibly leading to a higher mechanical failure rate through cracking.

Fig. 4 shows the normalized magnitude response of the disk while being vibrated from the shaker test fixture of Fig. 2 as the vibration frequency is swept. The y-axis in this plot is normalized to $38 \mu \mathrm{W}$. It can be seen here that the disk has multiple resonances, each with its own mode shape indicated by a small cartoon at each resonant peak. At the dominant mode near 1 


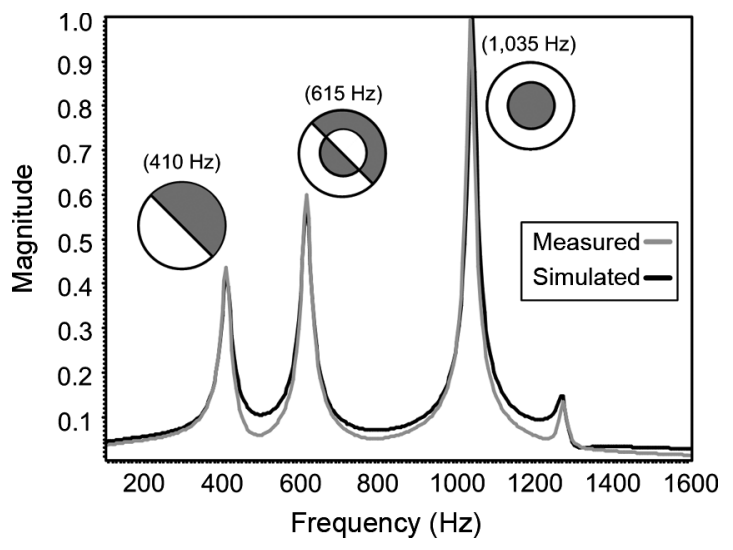

Fig. 4. Multiple resonances for piezoelectric disk transducer with multiple cuts in top plate electrode. Simulated results are from a lumped model of the disk derived using rigid-body analysis [3].

$\mathrm{kHz}$, the disk vibrates in a cupping motion, where all electrode signals will have the same phase. Near $400 \mathrm{~Hz}$ the disk vibrates in a folding motion, and at $615 \mathrm{~Hz}$ the desired traveling wave $(2,1)$ mode can be excited. The traveling wave is produced by exciting two modes which are orthogonal to each other. The magnitude response of the disk was also measured before the top plate was sectioned into multiple electrodes. Without the quarter-circle sections, the only mode for efficiently generating energy was near $1 \mathrm{kHz}$. The disk still vibrates at these other resonances, but the energy could not be harvested. The piezoelectric transducer will generate electrical charge proportional to the total integration of shearing force over its volume. With these lower frequency modes, some parts of the disk would have a positive shear force while others would have a negative shear force, and they would effectively cancel out each other. Sectioning the top-plate electrode allows separation of the positive charge from the negative charge, resulting in a net gain for energy harvesting at these lower frequency resonances.

\section{RECTIFIER}

In this section, we describe the design and optimization of a rectifier circuit for performing simple AC/DC conversion of the piezoelectric disk transducer's output. One goal of the design is to exploit the relative phase shift of the multiple electrodes to reduce the size of any output filter capacitor required to achieve a given voltage ripple specification. Fig. 5 shows a conventional (inductively loaded) MOS rectifier [8], which is commonly used for wireless power links. For the conventional rectifier, two large diode-connected PMOS devices, ML and MR, rectify the input voltage $\left(V_{i n}=V_{i n+}-V_{i n-}\right)$. Two small PMOS devices are used to bias the n-well of each large PMOS device. The positive feedback from the cross-coupled NMOS pair MN1-MN2 ensures that the more positive side of the AC input source, $V_{i n}$, will be connected to the load while the less positive side will be connected to ground. Initially, suppose $V_{\text {in }}>0 \mathrm{~V}>V_{\text {in- }}$ . Transistor MN1 turns on, clamping $V_{i n-}$ to ground and cutting off transistor MN2. Output $V_{\text {out }}$ tracks $V_{\text {in }}+$ through the power transistor on the right-hand side, MR. MR's bulk terminal is connected to $V_{i n+}$ through the small PMOS device

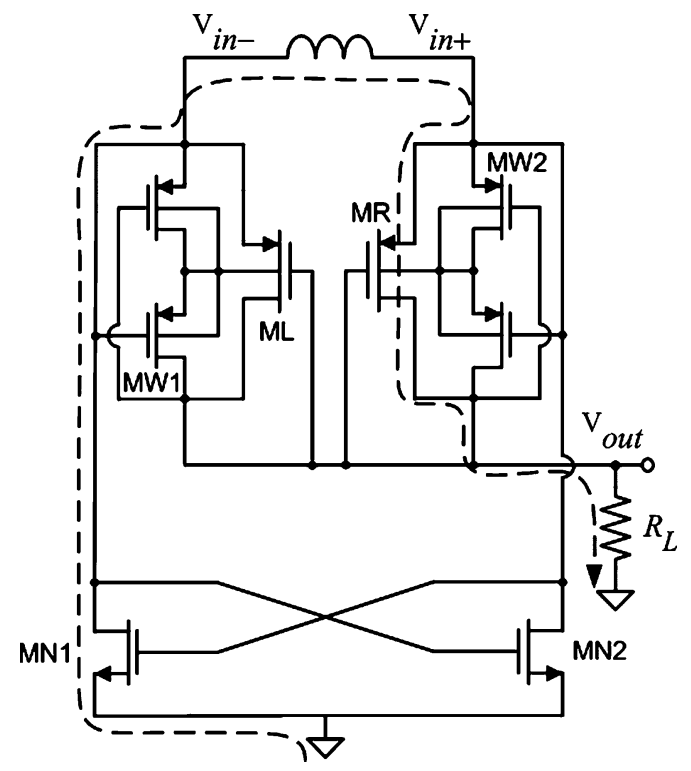

Fig. 5. Inductively loaded dual-input MOS regulator schematic. Devices MN1 and MN2 provide positive feedback, which ensures a current path from $V_{\text {in }}$ to both $V_{\text {out }}$ and ground.

MW2 since $V_{\text {out }}$ must be below $V_{\text {in }}$ by more than the magnitude of the threshold voltage of MR to ensure that MR is on and in the saturation region. This voltage drop ultimately limits the efficiency of the rectifier, since any load current must flow across the finite drain-source potential of MR and the associated power is essentially wasted. On the left-hand side, the bulk terminal of the other large PMOS transistor ML is connected to $V_{\text {out }}$ through the small PMOS MW1, since $V_{\text {out }}$ is higher than $V_{i n-}$. The dashed line in Fig. 5 shows the current path through the rectifier for this input condition. An analogous situation occurs when $V_{i n-}$ is positive, with each transistor on the left side of the circuit swapping roles with its corresponding device on the right.

Since the proposed piezoelectric disk has multiple electrodes, the less positive input does not need to be connected to ground in order to complete the loop for AC current flow, as it must in the conventional rectifier described above. Ground can instead be connected at a fixed common point on the disk such as the bottom plate.

The proposed rectifier designed for piezoelectric transducers is shown in Fig. 6. This dual-input piezoelectric rectifier consists of two CMOS-controlled rectifiers (CCRs) with their outputs connected together. The three transistors M1-M3 are identical in function to the three PMOS devices in half of the conventional rectifier above; however, here the gate of M1, instead of being shorted to the drain, is connected to the output of a CMOS inverter formed by transistors M4 and M5. In the proposed CCR, the body bias transistors M2-M3 also function as a crude peak detector, holding the well of M1 to the higher of $V_{\text {in }}$ and $V_{\text {out }}$. The well capacitance $C_{w}$ associated with the bulk of M1 helps to hold the peak voltage sampled through the body-bias transistors as the input voltage $V_{i n+}$ changes. The CMOS inverter M4-M5 functions as a low-power comparator basing its decision on the held peak-detector voltage and the CCR input voltage. The switching threshold of the inverter will 


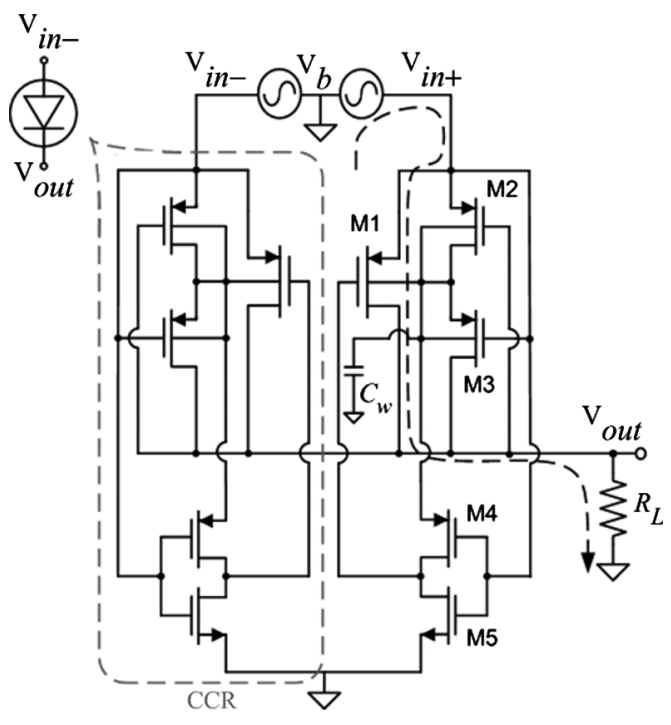

Fig. 6. Proposed piezoelectric dual-input rectifier schematic. It uses two CCRs. The dashed box encloses a single CCR. Ground is also connected to the bottom plate of piezoelectric disk.

be greater than the threshold voltage of M5 and depends on the relative sizing of M4 and M5. Body bias transistors M2-M3 ensure that the $p n$-junction associated with the n-well of the large PMOS power transistor M1 does not become forward biased [8]. Due to the low dropout voltage of the CCR, $V_{\text {out }}$ is near $V_{i n+}$ and thus M2 is off when M1 is on. The body of M1 is connected to $V_{i n+}$ through M2 before M1 turns on, and the body potential is held by the parasitic capacitor $C_{w}$ when M1 is on. Likewise, when the CCR turns off, the body of M1 connects to $V_{\text {out }}$ through M3. In addition, when M1 is off, M2 will also be off since $V_{\text {in }}$ is smaller than $V_{\text {out }}$.

Since the gate of M1 is effectively controlled by the voltage at its source, $V_{i n+}$, the phase relationship between $V_{i n+}$ and $V_{i n-}$ can be arbitrary, unlike the conventional rectifier where $V_{i n+}$ and $V_{i n-}$ must be $180^{\circ}$ out of phase to guarantee correct operation through the cross-coupled NMOS devices MN1-MN2. This allows the CCR to be used when rectifying the multiple output voltages of the piezoelectric disk transducer. For the proposed rectifier, the output voltage will be selected from the highest of the input voltages. In the conventional rectifier, the rectified output voltage is two times the input voltage when $V_{i n+}$ and $V_{i n-}$ are $180^{\circ}$ out of phase. A higher input voltage can be accomplished through stacking multiple piezoelectric transducers in series. For the prototype, each electrode can be associated with a single CCR, so four CCRs would be required for the quarter-circle electrodes in the proposed transducer. In addition, since the gate of M1 is not tied to its drain, M1 can be operated in the linear region, and the output voltage can follow the input without a threshold voltage drop. This significantly improves the voltage and power efficiency of the rectifier. The dark dashed line in Fig. 6 shows the current path from $V_{i n+}$ to ground through the CCR when M1 is on. Table I lists the drawn widths for the CCR prototype. All channel lengths are $0.35 \mu \mathrm{m}$.

The loss in the CCR will be dominated by a combination of the IR drop across the channel of M1 and the energy required
TABLE I

TRANSISTOR WIDTHS

\begin{tabular}{|l|c|}
\hline Transistor & Width \\
\hline M1 & $9 \mu \mathrm{m} \times 66$ \\
\hline M2 \& M3 & $9 \mu \mathrm{m} \times 6$ \\
\hline M4 & $9 \mu \mathrm{m} \times 3$ \\
\hline M5 & $9 \mu \mathrm{m} \times 3$ \\
\hline
\end{tabular}

All channel lengths are $0.35 \mu \mathrm{m}$.

to drive the gate of M1. The choice of the size of M1 will effectively trade off conduction loss for dynamic loss. An expression for the summation of loss terms (assuming a DC input voltage for the conduction loss and an $\mathrm{AC}$ input for the dynamic loss for simplicity) as a function of circuit parameters is given by

$$
\begin{aligned}
& \sum \text { Loss }=R_{\text {on }}\left[I_{\text {load }}\right]^{2}+(\text { Dynamic Loss }) \\
& \sum \text { Loss }=\left[\frac{\hat{V}_{\text {in }} \sqrt{g_{\text {on }}}}{1+g_{\text {on }} R_{\text {load }}}\right]^{2}+W C_{\text {gate }} \hat{V}_{\text {in }}^{2} f
\end{aligned}
$$

where $\hat{V}_{i n}$ is the peak input voltage, $R_{o n}=1 / g_{o n}$ is the on resistance of M1 in the triode region, $C_{\text {gate }}$ is the gate capacitance per unit width of M1, $W$ is the width of $\mathrm{M} 1$, and $f$ is the frequency of the input. As (2) suggests, the size of M1 is not critical, and widths from about $30 \mu \mathrm{m}$ to $150 \mu \mathrm{m}$ provide approximately the same efficiency. Keeping the losses below $5 \mu \mathrm{W}$ enables energy harvesting from parasitic mechanical vibrations.

For ultra-low power $(<1 \mathrm{~mW})$ systems, the rectifier efficiency and its equivalent loading on the piezoelectric transducer largely determine the total power available to the load. To maintain high conversion efficiency between mechanical and electrical energy, the CCR effectively implements a switched loading scheme on the vibrating transducer by open circuiting electrodes that fall below the threshold of the CMOS inverter (M4-M5). This scheme decreases the effective load as seen by the piezoelectric disk, allowing for increased peak voltage generation. Since the rectifier automatically switches between the peaks of the four input phases (each offset by $90^{\circ}$ of relative phase shift), an output filter capacitor is not needed to achieve modest output voltage ripple requirements. The exact ripple amount will depend on the load resistance, input vibration frequency and symmetry associated with the disk's electrodes and mounting apparatus.

\section{MeAsured Results}

Fig. 7 shows a block diagram of the test setup for the multiple-mode piezoelectric transducer with multiple-input rectifier. Four of the proposed CCRs from Fig. 6 are used to connect the four top-plate electrodes from the piezoelectric disk to the load $R_{L}$. An equivalent electrical model for each electrode is shown as the inset in Fig. 7. The disk is forced from its center by a mechanical shaker, which is in turn driven by an arbitrary waveform generator (AWG). In order to model typical environmental conditions during sensor operation, the AWG can be programmed to generate a pseudorandom waveform. 


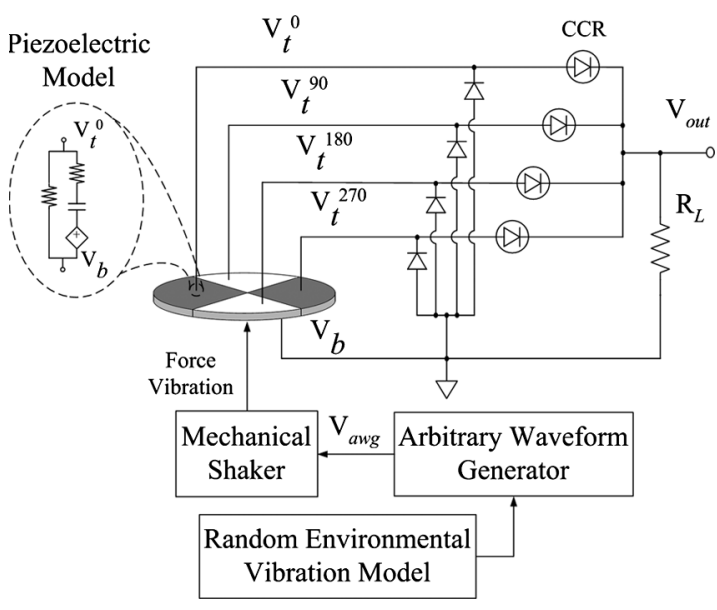

Fig. 7. Block diagram of test setup for random vibrational inputs. Piezoelectric disk has top-plate divided into four quarter-circle shaped electrodes and the bottom-plate intact. Top-plate electrodes are time-interleaved through CMOS diode switches.

As described above, the individual CCR thresholds are set by their own internal peak selector; the multiple-phase rectifier thus automatically adjusts to different input vibrations and electrode patterns without needing an additional power supply or clock. This allows for improved energy harvesting ability over a wider range of input vibration frequencies. The ESD protection diodes on each of the four CCR inputs provide the DC current path through the load. Several measurements on the proposed transducer and rectifier were performed by using the experimental apparatus shown in Fig. 7, including stimulating the disk with both sinusoidal and pseudorandom vibration patterns.

Fig. 8 shows measured waveforms from the proposed dual-input rectifier shown in Fig. 6. Snubbing diodes (shown in Fig. 7) that are used to protect against negative top plate voltages and to provide a DC current path cause the slight clipping visible at the negative voltage peaks. The rising transition of $V_{\text {out }}$ highlighted in the oscilloscope trace corresponds to the input voltage crossing the CMOS inverter switching threshold. After this transition, the output follows the input without a significant voltage drop. The inverter threshold can be changed by adjusting the relative sizes of M4 and M5. If the NMOS device M5 is made larger, then the CCR will turn on M1 at a lower input voltage. While shifting the threshold lower has the benefit of passing more total current (M1 is on over a larger fraction of the input half-cycle), with a lower threshold there is a greater chance of having more than one CCR on at a time, which may produce undesirable extra loading on the disk and consequently decrease the harvested energy.

Fig. 9 shows the measured waveforms from the multipleinput rectifier connected to the piezoelectric disk stimulated by the test fixture shown in Fig. 7. For the multiple-input rectifier, the vibration waveform, $V_{a w g}$, is shown along with a top-plate electrode signal, $V_{t}^{0}$, and the rectified output voltage, $V_{\text {out }}$. With the random input vibration, the rectifier can deliver $39 \mu \mathrm{W}$ of power to a $22 \mathrm{k} \Omega$ load. With a $30 \mathrm{nF}$ output capacitor and a $1 \mathrm{~V} 615 \mathrm{~Hz}$ input, the dual-input rectifier (shown in Fig. 6) and multiple-input rectifier (shown in Fig. 7) had measured peak-to-

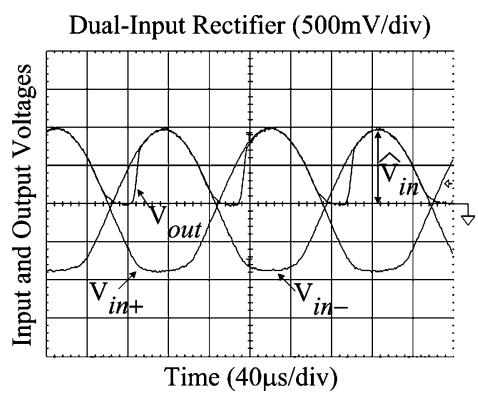

Fig. 8. Measured waveforms from the proposed dual-input rectifier from Fig. 6 driven with a $10 \mathrm{kHz}$ sinusoid.

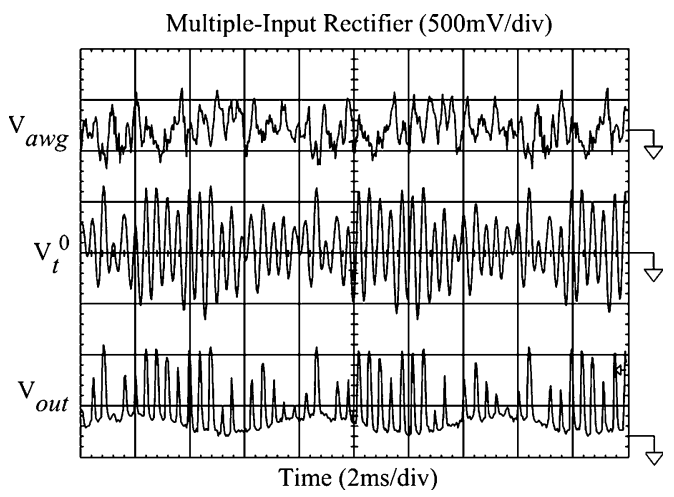

Fig. 9. Measured waveforms for the multiple-input rectifier stimulated by the test setup in Fig. 7.

peak output ripples of $440 \mathrm{mV}$ and $97 \mathrm{mV}$, respectively. When the traveling wave is excited in the disk, the additional electrode phases rectified by the multiple-input circuit help reduce the output ripple by more than a factor of four for a fixed output capacitance.

Fig. 10 shows a plot of the power efficiency for the conventional and proposed dual-input rectifiers versus load resistance. The proposed rectifier has a substantially better efficiency over a wide range of load conditions at the same peak input voltage. The power efficiency for the proposed rectifier is limited at low and high load resistances by conduction losses through M1 and the dynamic losses associated with the gate of M1, respectively. Fig. 11 shows the measured peak conversion ratio versus input amplitude for the proposed and conventional dual-input rectifiers. The conventional rectifier was implemented on a separate chip in $0.18 \mu \mathrm{m}$ CMOS as part of another project. Even with the advantages that the smaller feature size can provide, the proposed rectifier has a far superior conversion ratio to the conventional rectifier for peak input levels that are larger than the magnitude of a PMOS threshold voltage, which is about $0.7 \mathrm{~V}$ in the prototype.

Fig. 12 shows the measured output voltage for the multipleinput regulator, from Fig. 7, without an explicit output capacitor. The input vibration is a $615 \mathrm{~Hz}$ tone, which stimulates the traveling wave in the piezoelectric disk. Due to a slight imbalance in the mounting of the disk, the four electrode voltages are not equal in magnitude, causing a distorted waveform with a larger than expected ripple. The measured voltage ripple amplitude is roughly $35 \%$ of the peak rectified amplitude. In future designs, more accurate methods for scoring and mounting the disk will 


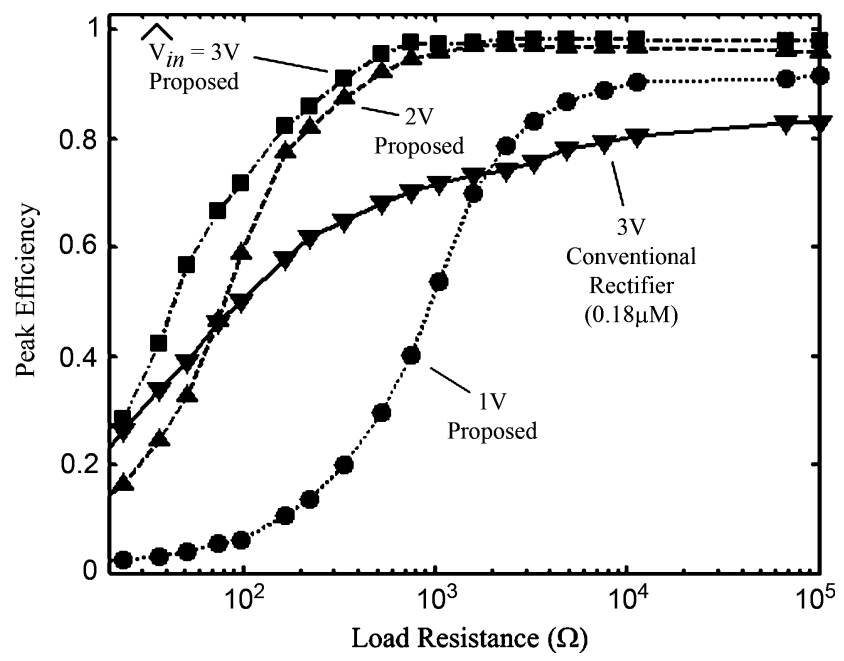

Fig. 10. Measured peak power efficiency over entire rectifier cycle versus load resistance for the conventional and proposed piezoelectric rectifiers with different peak input amplitudes $\hat{V}_{i n}$ and $f_{i n}=10 \mathrm{kHz}$. The conventional rectifier was built in $0.18 \mu \mathrm{m}$ CMOS.

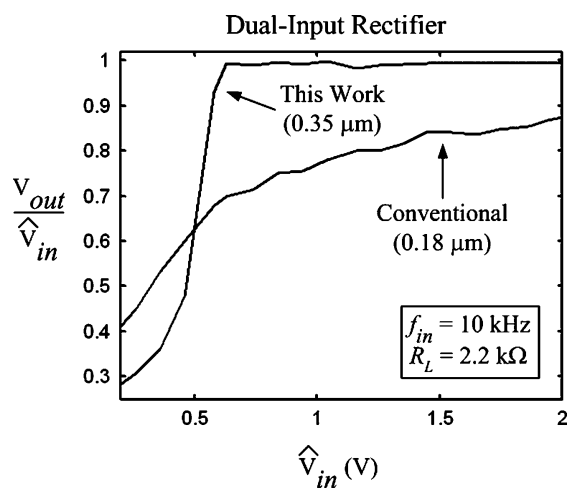

Fig. 11. Measured conversion ratios for conventional and proposed rectifiers. The proposed piezoelectric rectifier has superior performance for peak input voltages larger than a PMOS threshold.

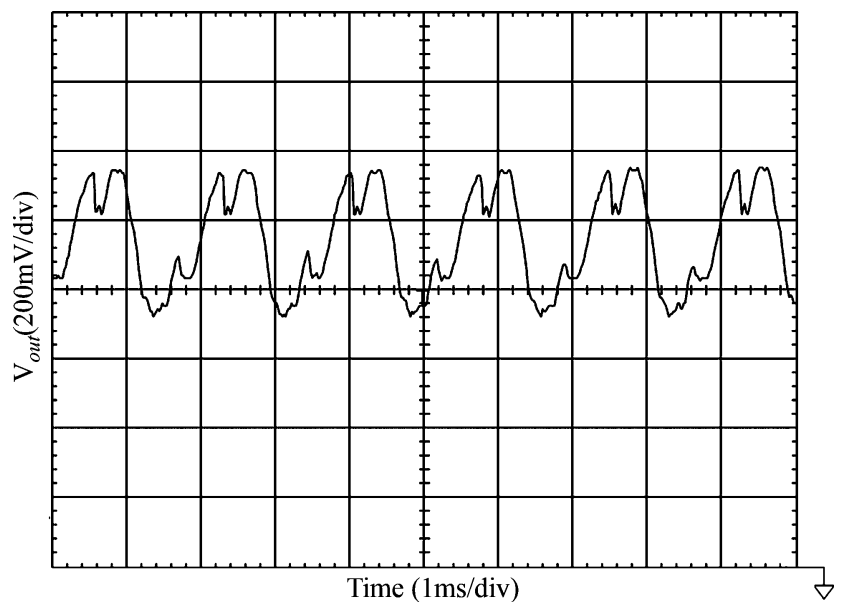

Fig. 12. Measured output voltage for the multiple-input rectifier with no explicit load capacitor. Input vibration is a $615 \mathrm{~Hz}$ tone, which stimulates the traveling wave in the piezoelectric disk.

produce better results. For example, the top-plate electrodes can be etched using standard processes.

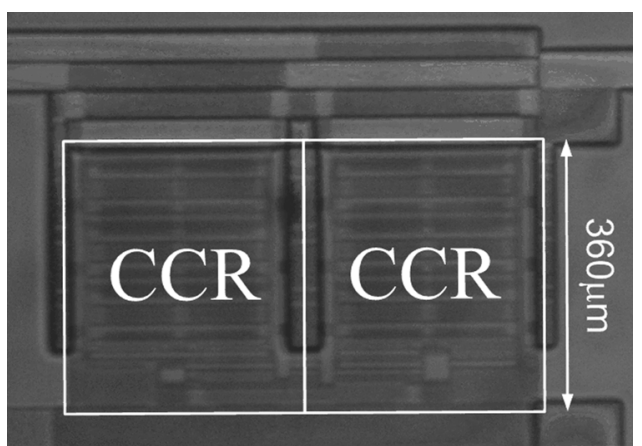

Fig. 13. Die photograph showing two CCRs, each $360 \mu \mathrm{m}$ on a side. Total PMOS power FET device width for each CCR $=500 \mu \mathrm{m}$.

TABLE II

COMPARISON OF MEASURED INTEGRATED CIRCUIT RESUlTS

\begin{tabular}{|l|c|c|c|c|c|c|c|c|}
\hline $\begin{array}{l}\text { Specification } \\
\text { (Unit) }\end{array}$ & $\begin{array}{c}\text { This } \\
\text { Work }\end{array}$ & {$[5]$} & {$[6]$} & {$[7]$} & {$[8]$} & {$[9]$} & {$[10]$} & {$[11]$} \\
\hline $\begin{array}{l}\text { CMOS } \\
\text { Technology ( } \mu \mathrm{m})\end{array}$ & 0.35 & 0.25 & 0.35 & 0.25 & 1.5 & 0.35 & 0.5 & 0.35 \\
\hline $\begin{array}{l}\text { Delivered Power } \\
(\mathrm{mW})\end{array}$ & 0.09 & 120 & 0.08 & 0.02 & 25 & 5 & 250 & 5.5 \\
\hline $\begin{array}{l}\text { Area } \\
\left(\mathrm{mm}^{2}\right)\end{array}$ & 0.007 & 3 & 0.008 & 0.015 & 0.48 & 0.0055 & 5.7 & 1.03 \\
\hline $\begin{array}{l}\text { Power Efficiency } \\
(\%)\end{array}$ & $\begin{array}{c}98.3 \\
(10 \mathrm{kHz})\end{array}$ & 87 & $36.6^{*}$ & 85 & 76 & 90 & 89 & 87.5 \\
\hline $\begin{array}{l}\text { Frequency Range } \\
(\mathrm{MHz})\end{array}$ & $<90$ & 0.67 & 953 & 0.35 & 10 & $<30$ & 2.5 & 1.5 \\
\hline $\begin{array}{l}\text { Drop Out Voltage } \\
(\mathrm{mV})\end{array}$ & 20 & N/A & N/A & 350 & 960 & 300 & 250 & 150 \\
\hline
\end{tabular}

A die photograph of the prototype is shown in Fig. 13.

\section{CONCLUSION}

This work has shown that the proposed CMOS rectifier can efficiently rectify the output voltages from a multiple-electrode piezoelectric transducer while being driven with a random vibration. Integrated peak selection circuitry provides an accurate and low power method for switching the CCRs. This enables efficient energy harvesting from many different vibrational modes, which may be stimulated simultaneously for random input vibrations. This flexibility allows for increased energy harvesting ability over a wide input vibration bandwidth. Table II compares measurements for this rectifier and other recent works. Previous works had power efficiencies between $76 \%$ and $90 \%$ while this work boasts a power efficiency greater than $98 \%$ with an input frequency of $10 \mathrm{kHz}$. For the application of wireless sensor nodes, it is desirable to have a rectifier that can deliver power on the order of microwatts. However, it is a significant challenge to deliver very small amounts of power while maintaining a high level of efficiency. The interface circuitry outlined in this paper has improved the state of the art in terms of efficiency and low drop-out voltage for low-frequency rectification and is applicable to numerous energy harvesting scenarios. 


\section{ACKNOWLEDGMENT}

The authors are grateful to Profs. S. Lewis, D. L. Margolis, and D. Horsley for valuable technical discussions.

\section{REFERENCES}

[1] A. Kansal and M. Srivastava, "An environmental energy harvesting framework for sensor networks," in Proc. IEEE Int. Symp. Low Power Electronics and Design (ISLPED'03), Aug. 2003, pp. 481-486.

[2] N. Guilar, R. Amirtharajah, and P. Hurst, "A full-wave rectifier for interfacing with multi-phase piezoelectric energy harvesters," in IEEE Int. Solid-State Circuits Conf. (ISSCC) Dig. Tech. Papers, Feb. 2008, pp. 302-303.

[3] N. Guilar, D. Margolis, D. Horsley, R. Amirtharajah, and P. Hurst, "Interface circuits for multiphase piezoelectric energy harvesters," in Proc. IEEE Applied Power Electronics Conf., Feb. 2008, pp. 639-644.

[4] G. Ottman, H. Hofmann, A. Bhatt, and G. Lesieutre, "Adaptive piezoelectric energy harvesting circuit for wireless remote power supply," IEEE Trans. Power Electron., vol. 17, no. 5, pp. 669-676, Sep. 2002.

[5] T. Y. Man, P. K. T. Mok, and M. Chan, "A CMOS-control rectifier for discontinuous-conduction mode switching DC-DC converters," in IEEE Int. Solid-State Circuits Conf. (ISSCC) Dig. Tech. Papers, Feb. 2006, pp. 358-359.

[6] N. Hiroyuki, H. Nakamoto, D. Yamazaki, T. Yamamoto, H. Kurata, S. Yamada, K. Mukaida, T. Ninomiya, T. Ohkawa, S. Masui, and K. Gotoh, "A passive UHF RFID tag LSI with $36.6 \%$ efficiency CMOSonly rectifier and current-mode demodulator in $0.35 \mu \mathrm{m}$ Feram technology," in IEEE Int. Solid-State Circuits Conf. (ISSCC) Dig. Tech. Papers, Feb. 2006, pp. 310-311.

[7] T. T. Le, J. Han, A. V. Jouanne, K. Mayaram, and T. S. Fiez, "Piezoelectric micro-power generation interface circuits," IEEE J. Solid-State Circuits, vol. 41, no. 6, pp. 1411-1420, Jun. 2006.

[8] M. Ghovanloo and K. Najafi, "Fully integrated wideband high-current rectifiers for inductively powered devices," IEEE J. Solid-State Circuits, vol. 39, no. 11, pp. 1976-1984, Nov. 2004.

[9] Y.-H. Lam, W.-H. Ki, and C.-Y. Tsui, "Integrated low-loss CMOS active rectifier for wirelessly powered devices," IEEE Trans. Circuits Syst. II, vol. 53, no. 12, pp. 1378-1382, Dec. 2006.

[10] E. Säckinger, A. Tennen, D. Shulman, B. Wani, M. Rambaud, D. Lim, F. Larsen, and G. S. Moschytz, "A 5-V AC-powered CMOS filterselectivity booster for POTS/ADSL splitter size reduction," IEEE J. Solid-State Circuits, vol. 41, no. 12, pp. 2877-2889, Dec. 2006.

[11] S. Guo and H. Lee, "An efficiency-enhanced integrated CMOS rectifier with comparator-controlled switches for transcutaneous powered implants," in Proc. IEEE Custom Integrated Circuits Conf. (CICC), Sep. 2007, pp. 385-388.

[12] M. M. Ahmadi and G. Jullien, "A full CMOS voltage regulating circuit for bioimplantable applications," in Proc. IEEE Midwest Symp. Circuits and Systems, 2005, vol. 2, pp. 988-991.

[13] M. Ghovanloo and N. Khalil, "A wideband frequency-shift keying wireless link for inductively powered biomedical implants," IEEE Trans. Circuits Sys. I, vol. 51, no. 12, pp. 2374-2383, Dec. 2004.

[14] S. Roundy, B. Otis, Y.-H. Chee, J. Rabaey, and P. Wright, "A 1.9 GHz RF transmit beacon using environmentally scavenged energy," in 2003 ISLPED Student Design Contest, Aug. 2003.

[15] A. Sinha, A. Wang, and A. Chandrakasan, "Energy scalable system design," IEEE Trans. Very Large Scale Integr. (VLSI) Syst., vol. 10, no. 2, pp. 135-145, Apr. 2002.

[16] T. Kenjo, Ultrasonic Motors: Theory and Design. Oxford, UK: Clarendon Press, 1993.

[17] S. Roundy, P. K. Wright, and J. Rabaey, Energy Scavenging for Wireless Sensor Networks with Special Focus on Vibrations. Boston, MA: Kluwer Academic.

[18] S. Roundy, E. Leland, J. Baker, E. Carleton, E. Reilly, E. Lai, B. Otis, J. Rabaey, V. Sundararajan, and P. K. Wright, "Improving power output for vibration-based energy scavengers," IEEE Pervasive Computing J. Mobile and Ubiquitous Computing, vol. 4, no. 1, pp. 28-36, Jan. 2005.

[19] E. Hong, S. Trolier-McKinstry, R. Smith, S. V. Krishnaswamy, and C. B. Freidhoff, "Vibration of micromachined circular piezoelectric diaphrams," IEEE Trans. Ultrason., Ferroelectr. Freq. Contr., vol. 53, no. 5, pp. 697-706, Apr. 2006.
[20] S. Platt, S. Farritor, and H. Haider, "On low-frequency electric power generation with PZT ceramics," IEEE/ASME Trans. Mechatron., vol. 10, no. 2, pp. 240-252, Apr. 2005.

[21] R. V. Southwell, "On the free transverse vibrations of a uniform circular disk clamped at its centre; and on the effects of rotation," Proc. Royal Society of London. Series A, Containing Papers of a Mathematical and Physical Character, vol. 101, no. 709, pp. 133-153, 1922.

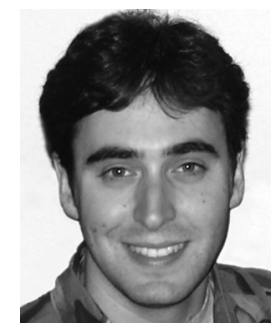

Nathaniel J. Guilar received the B.S. degree from Suffolk University, Boston, MA, in 2002, and the M.S. and Ph.D. degrees from the University of California at Davis in 2005 and 2008, respectively.

$\mathrm{He}$ joined Agilent Laboratories in 2008 at the Mixed-Signal Electronics Division in Santa Clara, CA, where he is currently working on high speed data converters and frequency synthesizers. His research interests include mixed-signal control systems, energy harvesting devices and analog circuit design.

Dr. Guilar has received the Accel Partners Fellowship, the GAANN Fellowship and the Analog Devices Outstanding Student Designer Award.

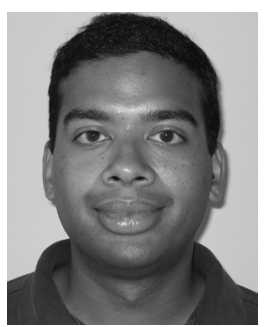

Rajeevan Amirtharajah received the S.B. and M.Eng. degrees in 1994, and the Ph.D. degree in 1999, all in electrical engineering from the Massachusetts Institute of Technology, Cambridge, MA. His doctoral work developed micropower DSP systems which scavenge energy from mechanical vibrations in their environment and use that energy to process information provided by embedded and wearable sensors.

From 1999 to 2002, as a senior member of the technical staff at High Speed Solutions Corp., Hudson, MA, later a subsidiary of Intel Corporation, he helped create innovative high performance multidrop bus technologies using electromagnetic coupling and pulse-based modulated signaling. He worked as an ASIC and mixed-signal circuit design consultant at SMaL Camera Technologies, Cambridge, MA, in 2003. In July 2003, he joined the Electrical and Computer Engineering Department at the University of California, Davis, where he is currently an Associate Professor. His research interests include low power VLSI design for sensor applications, powering systems from ambient energy sources, and high performance circuit and interconnect design.

Dr. Amirtharajah received the National Science Foundation CAREER award in 2006. He is an inventor on 18 U.S. patents and is a member of IEEE, AAAS, and Sigma Xi.

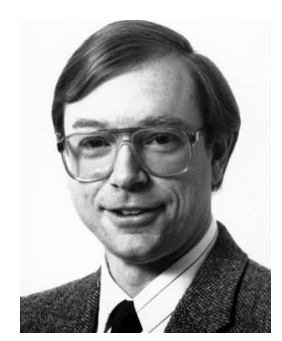

Paul J. Hurst (S'76-M'83-SM'94-F'01) received the B.S., M.S., and Ph.D. degrees in electrical engineering from the University of California at Berkeley in 1977,1979 , and 1983 , respectively.

From 1983 to 1984 , he was with the University of California, Berkeley, as a lecturer, teaching integrated-circuit design courses and working on an MOS delta-sigma modulator. In 1984, he joined Silicon Systems Inc., Nevada City, CA, where he was involved in the design of CMOS integrated circuits for voice-band modems. Since 1986, he has been on the faculty of the Department of Electrical and Computer Engineering at the University of California at Davis, where he is now a Professor. His research interests are in the areas of data converters and analog and mixed-signal integrated-circuit design for digital communications. He is a coauthor of a textbook on analog integrated-circuit design. He is also active as a consultant to industry.

Prof. Hurst has served on the program committees for the Symposium on VLSI Circuits and the IEEE International Solid-State Circuits Conference. He served for five years as an associate editor for the IEEE JOURNAL OF SOLIDStATE CIRCUITS. He is now a member of the administrative committee of the IEEE Solid-State Circuits Society. 